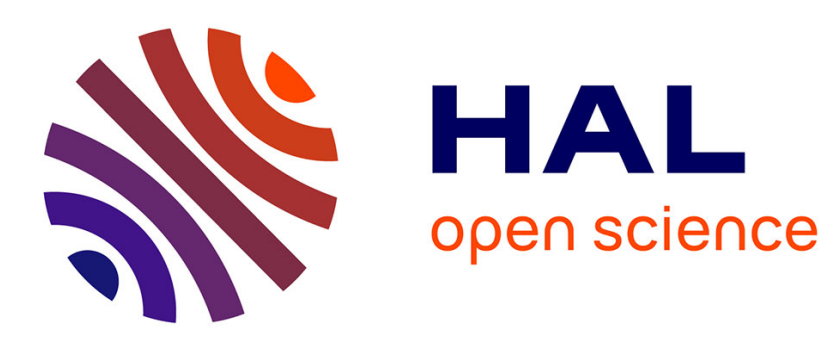

\title{
Relationship Between Blood Flow and Performance Recovery: A Randomized, Placebo-Controlled Study
}

Rachel Borne, Christophe Hausswirth, François Bieuzen

\section{To cite this version:}

Rachel Borne, Christophe Hausswirth, François Bieuzen. Relationship Between Blood Flow and Performance Recovery: A Randomized, Placebo-Controlled Study. International Journal of Sports Physiology and Performance, 2017, 12 (2), pp.152-160. 10.1123/ijspp.2015-0779 . hal-01734519

\section{HAL Id: hal-01734519 https://hal-insep.archives-ouvertes.fr/hal-01734519}

Submitted on 14 Mar 2018

HAL is a multi-disciplinary open access archive for the deposit and dissemination of scientific research documents, whether they are published or not. The documents may come from teaching and research institutions in France or abroad, or from public or private research centers.
L'archive ouverte pluridisciplinaire HAL, est destinée au dépôt et à la diffusion de documents scientifiques de niveau recherche, publiés ou non, émanant des établissements d'enseignement et de recherche français ou étrangers, des laboratoires publics ou privés. 
"Relationship Between Blood Flow and Performance Recovery: A Randomized, Placebo-Controlled Study" by Borne R, Hausswirth C, Bieuzen F

International Journal of Sports Physiology and Performance

(C) 2016 Human Kinetics, Inc.

Note. This article will be published in a forthcoming issue of the International Journal of Sports Physiology and Performance. The article appears here in its accepted, peer-reviewed form, as it was provided by the submitting author. It has not been copyedited, proofread, or formatted by the publisher.

Section: Original Investigation

Article Title: Relationship Between Blood Flow and Performance Recovery: A Randomized, Placebo-Controlled Study

Authors: Rachel Borne ${ }^{1,2}$, Christophe Hausswirth ${ }^{1}$, and François Bieuzen ${ }^{1}$

Affiliations: ${ }^{1}$ French National Institute of Sport (INSEP), Paris, France. ${ }^{2}$ Laboratory of Human Motricity, Education Sport and Health - EA 6312, University of Nice Sophia Antipolis, Nice, France.

Journal: International Journal of Sports Physiology and Performance

Acceptance Date: April 9, 2016

(C)2016 Human Kinetics, Inc.

DOI: $\underline{\text { http://dx.doi.org/10.1123/ijspp.2015-0779 }}$ 


\section{RELATIONSHIP BETWEEN BLOOD FLOW AND PERFORMANCE RECOVERY:}

\section{A RANDOMIZED, PLACEBO-CONTROLLED STUDY}

\section{(85 characters/85)}

\section{Submission type: Original investigation}

Authors: Rachel Borne ${ }^{1,2}$, Christophe Hausswirth ${ }^{1}$, François Bieuzen ${ }^{1}$

Affiliations: ${ }^{1}$ French National Institute of Sport (INSEP), Paris, France

${ }^{2}$ Laboratory of Human Motricity, Education Sport and Health - EA 6312, University of Nice Sophia Antipolis, Nice, France

\section{Corresponding author:}

François BIEUZEN, $P h D$

French National Institute of Sport (INSEP)

11 avenue du Tremblay, 75012 Paris, France

f.bieuzen@icloud.com

Tel: +33-1.41.74.41.65

Preferred running head: Blood flow and performance recovery (36 char/40) 
"Relationship Between Blood Flow and Performance Recovery: A Randomized, Placebo-Controlled Study" by Borne R, Hausswirth C, Bieuzen F

International Journal of Sports Physiology and Performance

(C) 2016 Human Kinetics, Inc.

\begin{abstract}
Purpose: This study investigated the effect of different limb blood flow levels on cycling performance recovery, blood lactate concentration and heart rate. Methods: Thirty-three highintensity intermittent trained athletes completed two $30 \mathrm{~s}$ Wingate anaerobic test sessions: $3 \mathrm{x}$ $30 \mathrm{~s}$ Wingate test (WAnT 1-3) and 1 x $30 \mathrm{~s}$ Wingate test (WAnT 4), on a cycling ergometer. The two Wingate test sessions (WAnT 1-3 and WAnT 4) were separated by a randomly assigned 24 min recovery intervention selected from among: blood flow restriction, passive rest, placebo stimulation or neuromuscular electrical stimulation-induced blood flow. Calf arterial inflow was measured by venous occlusion plethysmography at regular intervals throughout the recovery period. Performance was measured in terms of peak power and mean power output during the first (WAnT 1) and the fourth (WAnT 4) Wingate test. Results: Following the recovery interventions, a large $(\mathrm{r}=0.68$ (90\% CL $0.42 ; 0.83))$, and very large $(\mathrm{r}$ $=0.72(90 \%$ CL $0.49 ; 0.86))$ positive correlation was observed between the change in calf arterial inflow and the change in mean power and peak power output, respectively. Calf arterial inflow was significantly higher during the neuromuscular electrical stimulation recovery intervention compared to the blood flow restriction, passive rest and placebo stimulation interventions $(\mathrm{P}<0.001)$. This corresponds to the only intervention that allowed performance recovery $(\mathrm{P}>0.05)$. No recovery effect was linked to heart rate or blood lactate levels. Conclusions: For the first time, these data support the existence of a positive correlation between an increase in blood flow and performance recovery between bouts of high-intensity exercise. As a practical consideration, this effect can be obtained by using neuromuscular electrical stimulation-induced blood flow since this passive, simple strategy could be easily applied during short-term recovery.
\end{abstract}

Key words: limb blood flow level; high-intensity exercise; neuromuscular electrical stimulation; short-term recovery 
"Relationship Between Blood Flow and Performance Recovery: A Randomized, Placebo-Controlled Study"

by Borne R, Hausswirth C, Bieuzen F

International Journal of Sports Physiology and Performance

(C) 2016 Human Kinetics, Inc.

\section{Introduction}

In many sporting situations it is essential to be able to perform and recover rapidly from high-intensity bouts of exercise. As an example, in sprint track cycling, a number of maximal effort performances are necessary in a short period of time. Each bout of high-intensity intermittent exercise results in fuel degradation and metabolic perturbations inducing transitory fatigue which can limit optimal sporting performance ${ }^{1}$. Higher levels of fatigue may also predispose athletes to tissue injury or non-functional overreaching. It therefore appears necessary to assist the return to a pre-exercise state for physiological systems in preparation for subsequent exercise. Recovery can be enhanced by applying an appropriate recovery strategy 2,3 .

To improve high-intensity short-term recovery, many strategies have been suggested. The most frequently proposed mechanism through which strategies may contribute to recovery after high-intensity exercise is by increasing or maintaining local and/or systemic blood flow ${ }^{4-}$ ${ }^{8}$. Effective recovery strategies enhance blood flow to the recovering muscles, which is assumed to improve oxygen and nutrient delivery, and therefore $\mathrm{PCr}$ and glycogen resynthesis 9 , while at the same time transporting lactate and metabolic by-products such as $\mathrm{H}^{+}$ions, $\mathrm{Pi}, \mathrm{Mg}^{2+}$, and $\mathrm{NH}_{3}$ away from the active muscles to removal sites and non-exercised muscles ${ }^{10}$.

The hemodynamics of arterial circulation are relatively simple to understand; they are dominated by the pumping function of the heart. In contrast, the hemodynamics of venous return in the lower limbs, against gravity, are more complicated and multifactorial, and tend to be dominated by the role played by the contracting calf muscle ${ }^{11}$. According to some studies, an increase in local and/or systemic blood flow can be achieved through several parameters such as activation of the skeletal muscle pump and release of endothelium-derived relaxing factors which increase venous return and arterial inflow ${ }^{12,13}$. However, it remains unclear whether greater blood flow immediately after exercise improves short-term recovery 
"Relationship Between Blood Flow and Performance Recovery: A Randomized, Placebo-Controlled Study"

by Borne R, Hausswirth C, Bieuzen F

International Journal of Sports Physiology and Performance

(C) 2016 Human Kinetics, Inc.

and performance during subsequent high-intensity exercise. Moreover, no study has yet established to what extent the increased blood flow level is sufficient to benefit subsequent performance.

Neuromuscular electrical stimulation (NMES) is a means of increasing blood flow to the muscles through low-frequency stimulation ${ }^{14,15}$. NMES is used in clinical settings as an effective non-pharmacological means to treat vascular disorders. Involuntary muscle contractions effectively increase lower limb blood flow via the "muscle pump effect" and trigger the release of vasoactive substances ${ }^{16}$. Theoretically, this technique could produce some of the benefits expected from active post-exercise recovery ${ }^{17}$. NMES could also have the advantage of not impairing the replenishment of muscle $\mathrm{PCr}$ and glycogen stores as it limits metabolic demands relative to active recovery ${ }^{18}$. Recent scientific evidence indicates that NMES can be considered to be an effective method of enhancing performance recovery compared to alternative methods, in both untrained and athletic populations ${ }^{17,19-22}$.

As suggested by Broatch, et al. ${ }^{23}$ in a recent paper, one major problem with testing recovery modalities is the placebo effect. Indeed, several works focusing on recovery of sporting performance fail to include a placebo situation even though the placebo effect has been extensively described in medicine. The placebo effect influences both sporting performance $^{24}$ and performance recovery ${ }^{23}$. In light of these effects, it is crucial to incorporate a placebo condition in any study.

The aim of the present study was to investigate the influence of different limb blood flow levels, blood flow restriction, passive recovery, placebo protocol and NMES-induced blood flow, applied for the entire duration of the short-term recovery period following a model high-intensity sprint exercise. 
"Relationship Between Blood Flow and Performance Recovery: A Randomized, Placebo-Controlled Study"

by Borne R, Hausswirth C, Bieuzen F

International Journal of Sports Physiology and Performance

(C) 2016 Human Kinetics, Inc.

\section{Methods}

\section{Participants}

33 sprinters in cycling and middle-distance runners participated in this study (Table 1). All undertook a minimum of three vigorous exercise sessions per week. After being informed of the experimental procedures and potential risks, all completed the written informed consent. The experiment was conducted in compliance with the Declaration of Helsinki and was approved by the local ethics committee.

Participants were asked to eat as normal (at least $3 \mathrm{~h}$ before the test), to stay well hydrated and to abstain from high-intensity physical activities for at least $24 \mathrm{~h}$ prior to each session. They also asked to refrain from smoking and consuming drinks containing caffeine and alcohol on the day of each session.

\section{Experimental design}

Participants first familiarized with the test protocol to eliminate the practice/learning effects of the protocol. On a subsequent visit, they completed three standard $30 \mathrm{~s}$ Wingate anaerobic test (WAnT 1-3), with the purpose of inducing extreme fatigue prior to a randomly assigned $30 \mathrm{~min}$ recovery intervention protocol, including $24 \mathrm{~min}$ of either: blood flow restriction (BFR), passive recovery (PAS), placebo stimulation (NMES Placebo $_{\text {) }}$ or Neuromuscular electrical stimulation-induced blood flow $\left(\mathrm{NMES}_{\mathrm{BF}+}\right)$. After the recovery period, participants completed a single standard $30 \mathrm{~s}$ Wingate test (WAnT 4) as the postintervention exercise session. An overview of the experimental design is presented figure 1.

\section{Wingate test protocols}

Participants performed a 5 min warm-up, which consisted of cycling on an electromagnetically braked cycle ergometer (Excalibur sport, Lode BV, Groningen, the Netherlands) at $80 \mathrm{~W}$, at $80 \mathrm{RPM}$ cadence. This also included a $5 \mathrm{~s}$ acceleration at the end of 
"Relationship Between Blood Flow and Performance Recovery: A Randomized, Placebo-Controlled Study"

by Borne R, Hausswirth C, Bieuzen F

International Journal of Sports Physiology and Performance

(C) 2016 Human Kinetics, Inc.

the second minute. Participants then performed 3 x $30 \mathrm{~s}$ WAnT in sequence, interspersed by 4 min of cycling at $80 \mathrm{~W}$ at $80 \mathrm{RPM}$. After the recovery period, a single standard $30 \mathrm{~s}$ WAnT (WAnT 4), including the 5 min warm-up procedure, was completed as the post-intervention exercise session. For each $30 \mathrm{~s}$ WAnT the braking force applied was $7 \%$ body mass for men and $6.5 \%$ for women ${ }^{25}$. Participants were encouraged to cycle "all-out" at the highest cadence possible for the duration of the $30 \mathrm{~s}$ test period and to reach their maximal power as soon as possible. They were instructed to grip the handlebars and to not lift off the saddle while cycling. During each bout the cycle ergometer was controlled via a PC running Lode Wingate software (version 1.0.12, lode BV, Groningen, the Netherlands). Average data for peak power (PPO) and mean power (MPO) output values were recorded over the first (WAnT 1) and the fourth (WAnT 4) Wingate test.

\section{Recovery interventions}

Immediately after the first session (WAnT 1-3), participants were randomly assigned to the 30 min recovery intervention protocol, including a 3 min transition from the end of WAnT 1-3 to the setting up of the plethysmograph and skin electrodes or elastic wraps, followed by a 24 min treatment period, then a further 3 min transition period back to the ergometer to complete WAnT 4. For all conditions, including BFR, NMES $S_{\text {Placebo }}$ and passive conditions, the participants were lead to believe that the proposed recovery modality was beneficial in recovery from high-intensity exercise.

Participants in the NMES-induced blood flow $\left(\mathrm{NMES}_{\mathrm{BF}+}\right)$ condition used a commercially available electrical stimulator of the calf muscles (Veinoplus ${ }^{\circledR}$ v.2.1, Ad Rem Technology, Paris, France). The stimulation was applied via two self-adhesive Veinopack $8 \mathrm{x}$ $13 \mathrm{~cm}$ surface electrodes (Ad Rem Technology, Paris, France). The electrodes were placed on the medio-central part of the calf, with one electrode for one leg of each participant. It has been 
"Relationship Between Blood Flow and Performance Recovery: A Randomized, Placebo-Controlled Study"

by Borne R, Hausswirth C, Bieuzen F

International Journal of Sports Physiology and Performance

(C) 2016 Human Kinetics, Inc.

established that this stimulation technology is an effective method of activating the calf muscle pump and enhancing arterial and venous limb blood flow, in healthy individuals and peripheral vascular disease patients ${ }^{14,15}$. The stimulation pattern and application recommendations are described by Bieuzen, et al. ${ }^{17}$ and Cywinski ${ }^{26}$. A minimal threshold was fixed by the investigators corresponding to a visible contraction of the calf muscles (to act as a muscle pump) with comfortable sensation.

In the NMESPlacebo condition, the placebo stimulator was physically identical to the real Veinoplus ${ }^{\circledR}$ v.2.1, excepted that the output signal was similar to a weak transcutaneous electrical nerve stimulation and not to the NMES stimulus deliverable by the real Veinoplus ${ }^{\circledR}$. In effect, the placebo stimulator produced a delicate and continuous tingling sensation on the skin, but it was too weak to induce rhythmic and visible contractions of the participant calf muscles.

The blood flow restriction (BFR) was applied with elastic wraps (width, $7 \mathrm{~cm}$; length, $80 \mathrm{~cm}$ ) that were placed to the upper part of each thigh. They were tightened at a pressure of $90-110 \mathrm{~mm} \mathrm{Hg}$ interval and maintained at this pressure throughout the $24 \mathrm{~min}$ of recovery intervention period. The elastic pressure of $90-110 \mathrm{~mm} \mathrm{Hg}$ interval was selected because the occlusive stimulus at this pressure has been suggested to restrict venous blood flow and cause pooling of blood in capacitance vessels distal to the cuff, ultimately restricting arterial blood flow $^{27}$. If it is not possible to create a BFR placebo condition, the participants were lead to believe that a compression, with elastic wraps, was beneficial in recovery from high-intensity exercise.

In the passive condition (PAS), participants were placed in the same lying position as for $\mathrm{BFR}, \mathrm{NMES} S_{\text {Placebo }}$ and $\mathrm{NMES}_{\mathrm{BF}+}$ recovery interventions and were instructed to observe passive rest for $24 \mathrm{~min}$. 
"Relationship Between Blood Flow and Performance Recovery: A Randomized, Placebo-Controlled Study"

by Borne R, Hausswirth C, Bieuzen F

International Journal of Sports Physiology and Performance

(C) 2016 Human Kinetics, Inc.

\section{Measurements}

Calf arterial inflow in the left leg was measured by using venous occlusion plethysmography ${ }^{16}$. An inflating cuff (SC10D, Hokanson, Bellevue, USA) was placed around the participant's thigh (below the elastic wrap) to occlude venous blood flow and connected to a rapid cuff inflator (E20, Hokanson), which was set above venous, but below arterial pressure (50 $\mathrm{mmHg}$ ). A suitable strain gauge (Hokanson) was placed around the widest part of the calf (above the NMES electrode) and connected to a plethysmograph (EC6, Hokanson). Participants lay in a supine position with their legs supported $10 \mathrm{~cm}$ above heart level to empty the venous system. To exclude the foot circulation, a segmental pressure cuff (TMC7, Hokanson) was placed around the ankle and inflated to $50 \mathrm{mmHg}$ pressure at the same time as the thigh cuff. Data were recorded on a laptop using the NIVP3 arterial inflow studies software (Hokanson). On commencing testing, the cuffs were inflated to $50 \mathrm{mmHg}$ for $5 \mathrm{~s}$ at a time. During each $5 \mathrm{~s}$ occlusion interval, changes in calf circumference were detected as changes in electrical resistance in the strain gauge. The rate of change was interpolated to represent change in limb volume by using the unit $\mathrm{ml}$ per $100 \mathrm{ml} / \mathrm{min}$. Five readings were taken at rest and one reading was at regular interval throughout the 30 min recovery period (i.e. minutes $4,6,9,13$, 17, 21, 25 from the end of WAnT 1-3). In the NMES ${ }_{\mathrm{BF}+}$ and NMES Placebo conditions, the measures realized during the recovery intervention period needed the electrodes cable unplugging during $5 \mathrm{~s}$ but not the power supply allowing the preservation of the device pulse sequence.

Blood lactate and heart rate were considered at rest and at regular interval throughout the 30 min recovery period (i.e. minutes $1,4,6,9,13,17,21,25$ from the end of WAnT 1-3). Blood lactate concentrations from capillary finger samples $(20 \mu \mathrm{l})$ were collected and analyzed with a Biosen Lactate analyzer (Biosen C-line analyzer, EKF Industrie, Elektronik GmbH, 
"Relationship Between Blood Flow and Performance Recovery: A Randomized, Placebo-Controlled Study"

by Borne R, Hausswirth C, Bieuzen F

International Journal of Sports Physiology and Performance

(C) 2016 Human Kinetics, Inc.

Barbelen, Germany). Heart rate was recorded every $5 \mathrm{~s}$ throughout all experimental protocol (Polar RS400, Polar, Kempele, Finland).

Finally, participants were asked to evaluate the recovery intervention (efficacy: « how recovered do you feel after this recovery intervention? »; well-being: «how did you like this recovery intervention? ») by means of a 10 points visual analogue scale, ranging from " not at all”' (0 point) to "very, very much”'(10 points $)^{28}$.

\section{Statistical analyses}

We assessed the normality of each variable from a normal probability plot and confirmed the distribution using the Kolmogorov-Smirnov statistic. Homogeneity of variance was assessed with Levene's test. Each of these analyses was performed using $\operatorname{SPSS}^{\circledR} 20.0$ software (SPSS, Chicago, Illinois, USA). Of note, statistical analysis was performed on 31 complete data sets because one participant of the $\mathrm{NMES}_{\mathrm{BF}+}$ condition did not complete one of its performance bouts and equipment failure occurred during one of the bouts for another participant of the NMES Placebo condition.

A two-way (recovery $\times$ time) repeated measures analysis of variance (ANOVA) was used to examine differences in dependent variables (i.e. mean power and peak power output, calf arterial inflow, blood lactate concentration, heart rate and perceptions of recovery) between recovery intervention means at each time point. If a significant main effect was observed, pairwise comparisons were conducted using Bonferonni post-hoc analysis. For these analyses, significance was accepted at $\mathrm{P}<0.05$.

The relationships between the change in performance (mean power and peak power output) and the change in blood flow were assessed by analysis of individual response. For each individual, the difference in response to a given variable was calculated as a percentage of the individual response between the first (WAnT 1) and the fourth (WAnT 4) Wingate test 
"Relationship Between Blood Flow and Performance Recovery: A Randomized, Placebo-Controlled Study"

by Borne R, Hausswirth C, Bieuzen F

International Journal of Sports Physiology and Performance

(C) 2016 Human Kinetics, Inc.

(i.e., mean power and peak power output) for performance and between the rest, before the testing session, and the recovery intervention period (i.e. $24 \mathrm{~min}$ ) for calf arterial inflow. A Pearson's product-moment correlation analysis was used to examine the relationship between the change in performance, mean or peak power, and the change in blood flow. The following criteria were adopted to interpret the magnitude of correlation (r) between test measures: $\leq 0.1$, trivial $;>0.1-0.3$, small $;>0.3-0.5$, moderate $;>0.5-0.7$, large $;>0.7-0.9$, very large $;>0.9-1$, almost perfect ${ }^{29}$. If the confidence limits overlapped zero, the outcome was considered unclear. Data are presented as means $\pm \mathrm{SD}$, unless otherwise stated.

\section{Results}

\section{Calf arterial inflow}

The variation of calf arterial inflow expressed as a percentage of the pre intervention measurement is presented in Table 2. Analysis of variance revealed a significant effect for recovery type $\left(F_{3,29}=52.86, P<0.001\right)$, time effect $\left(F_{2,58}=9.74, P<0.001\right)$ and recovery type $\mathrm{x}$ time interaction $\left(F_{6,58}=2.47, P=0.03\right)$. Post-hoc analysis indicated that calf arterial inflow was significantly higher during the $\mathrm{NMES}_{\mathrm{BF}+}$ recovery intervention compared to the BFR, PAS and NMES $S_{\text {Placebo }}$ recovery interventions $(\mathrm{P}<0.001)$. Calf arterial inflow was significantly lower during the BFR recovery intervention compared to the PAS and NMES $S_{\text {Placebo }}$ recovery interventions $(\mathrm{P}<0.001)$. Post-hoc analysis indicated that calf arterial inflow was significantly decreased at the end of of the recovery intervention period (21-25 min) compared to the start of this period (4-9 min) only in the $\mathrm{NMES}_{\mathrm{BF}+}$ recovery intervention $(\mathrm{P}<$ $0.001)$.

\section{Performance}

Table 2 details the sum of the total work measured during the first Wingate anaerobic test session (WAnT 1-3), the mean power (MPO) and peak power (PPO) output values of the 
"Relationship Between Blood Flow and Performance Recovery: A Randomized, Placebo-Controlled Study"

by Borne R, Hausswirth C, Bieuzen F

International Journal of Sports Physiology and Performance

(C) 2016 Human Kinetics, Inc.

first (WAnT 1) and the fourth (WAnT 4) Wingate test. The sum of the total work during WAnT 1-3 was not different between groups $\left(F_{3,27}=0.007, P=0.99\right)$. Analysis of variance of the mean power (MPO) and peak power (PPO) output values revealed a significant effect for time $\left(F_{1,27}=107.31, P<0.001\right)$ and a significant recovery type $\mathrm{x}$ time interaction $\left(F_{3,27}=10.64, P\right.$ $<0.001)$. However, there was no recovery effect $\left(F_{3,27}=10.64, P=0.40\right)$. Post-hoc analysis indicated that PPO and MPO output values were impaired at WAnT 4 compared with WAnT 1 when BFR $(\mathrm{P}<0.001)$, PAS $(\mathrm{P}<0.001)$ and NMES ${ }_{\text {Placebo }}(\mathrm{P}<0.05)$ recovery interventions were used.

\section{Relationship between calf arterial inflow and subsequent exercise performance}

The relationships between the change in calf arterial inflow (between rest and 24 min recovery intervention period) and the change in mean power (A) and peak power (B) output values (between WAnT 1 and WAnT 4) are presented in figure 2. Pearson's product-moment correlation analysis revealed a large $(\mathrm{r}=0.68(90 \%$ CL $0.42 ; 0.83))$, and a very large $(\mathrm{r}=0.72$ (90\% CL $0.49 ; 0.86)$ ) correlation between the change in calf arterial inflow and the change in mean power and peak power output, respectively.

\section{Blood lactate and heart rate}

Data for blood lactate concentration and heart rate are presented in figure 3. Analysis of variance revealed a significant time effect (blood lactate, $F_{7,21}=616.26, P<0.001$ and heart rate, $\left.F_{7,21}=118.34, P<0.001\right)$ but no recovery effect $\left(F_{3,27}=1.02, P=0.40\right.$ and $F_{3,27}=0.25$, $P=0.86$, respectively $)$ and no recovery type $\mathrm{x}$ time interaction $\left(F_{7,21}=0.99, P=0.48\right.$ and $F_{7,21}$ $=1.09, P=0.37$, respectively).

\section{Perceived recovery}

Table 3 details the mean recovery perceptions at the end of the recovery intervention

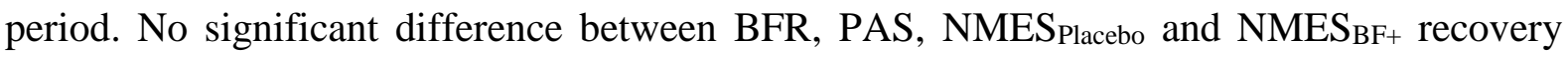


"Relationship Between Blood Flow and Performance Recovery: A Randomized, Placebo-Controlled Study"

by Borne R, Hausswirth C, Bieuzen F

International Journal of Sports Physiology and Performance

(C) 2016 Human Kinetics, Inc.

interventions was observed, based on the scores awarded by participants on the two visual analogue scales (efficacy and well-being) (P > 0.05).

\section{Discussion}

To our knowledge, this is the first study to compare the effects of applying different limb blood flow levels during recovery after exhausting exercise. From our data, $\mathrm{NMES} \mathrm{BF}_{\mathrm{B}+}$, inducing a $\sim 243 \% \pm 76 \%$ calf arterial inflow increase between rest and recovery intervention period, is of greater benefit to repeat performance than interventions involving blood flow restriction (BFR), passive recovery (PAS) or placebo stimulation (NMES Placebo). This outcome is supported by several recent studies demonstrating better performance following NMES protocols $^{17,19-22}$. However, methodological aspects of NMES can significantly alter its impact on performance. The aspects known to be influential include the stimulation intensity selected, the frequency modulations and the location to which stimulation is applied. In addition, the efficacy of this recovery strategy also depends on the duration of stimulation during each phase, the total treatment time relative to the total recovery time, and the profile of the performance test. None of these appear to have been examined previously in a systematic manner. Moreover, even if a minimal threshold was set by investigators, corresponding to a visible contraction of the calf muscles with comfortable sensation, there appears to be a certain degree of individual variability in sensitivity to blood flow response with $\mathrm{NMES}_{\mathrm{BF}+}$ (values ranging from $161 \% \pm$ $49 \%$ to $386 \% \pm 77 \%$ calf arterial inflow increase for the variation between rest and recovery intervention period). As an example, Griffin et al. (2010) in their study reported gender as a clinical factor affecting efficacy of $\mathrm{NMES}_{\mathrm{BF}+}$ on leg venous flow, in healthy individuals. Indeed, male gender was associated with a higher peak systolic velocities and higher values of ejected total volume per minute, in the popliteal veins. Therefore, further research is needed to identify the most appropriate blood flow stimulation level for performance recovery, depending 
"Relationship Between Blood Flow and Performance Recovery: A Randomized, Placebo-Controlled Study"

by Borne R, Hausswirth C, Bieuzen F

International Journal of Sports Physiology and Performance

(C) 2016 Human Kinetics, Inc.

on recovery period and exercise modes. This could elucidate the relative importance of the blood flow modulation in improving exercise performance.

Interestingly, a $66 \% \pm 57 \%$ and $71 \% \pm 37 \%$ calf arterial inflow increase during recovery intervention period, compared to baseline values, was noted with the passive and placebo protocols, respectively. This increase in blood flow is a physiological response reflecting exercise-induced reactive hyperemia. Indeed, an enormous increase in blood flow to the skeletal muscle is necessary during exercise to meet the 20 to 50 fold increase in oxygen and substrate consumption required to support the significant increase in metabolic activity within the active muscles 9 .

Notwithstanding limb blood flow level differences induced by the four recovery interventions, no significant difference was noted between BFR, PAS, NMESPlacebo and $\mathrm{NMES}_{\mathrm{BF}+}$ on heart rate, during the 24 min intervention period. In one hand, we suggest that the first exercise session, corresponding to a very exhaustive exercise, could have induced an important cardiac demand, needing a long recovery period to return to baseline. Indeed, Malone, et al. ${ }^{8}$ in their study have realized the exact same exercise-induced fatigue protocol ( 3 x $30 \mathrm{WAnT})$. They found similar results regarding heart rate during a $30 \mathrm{~min}$ recovery period of passive or NMES induced-blood flow recovery intervention. In a second hand and based of the Franck-Starling law of the heart, we hypothesize that the four recovery interventions could have influenced preload, stroke volume and cardiac output, without having an influence on heart rate. Finally, as heart rate is a parameter with very individual response regard rest and exercise, a parallel study could have made differences greater between recovery interventions. Further investigation is needed to clarify the physiological impact of the limb blood flow modulation on the cardiac parameters.

When considering $\mathrm{NMES}_{\mathrm{BF}+}$ as an aid to recovery between repeated high-intensity exercise sessions, several effects are expected which could potentially explain the improvement 
"Relationship Between Blood Flow and Performance Recovery: A Randomized, Placebo-Controlled Study"

by Borne R, Hausswirth C, Bieuzen F

International Journal of Sports Physiology and Performance

(C) 2016 Human Kinetics, Inc.

in performance recovery observed with the blood flow stimulation protocol used here. The first effect is an acceleration of the removal of metabolic by-products from the muscle through an increase in or maintenance of local and/or systemic blood flow. Maintaining adequate blood flow to the recovering muscles will allow clearance of the accumulated lactic acid and other metabolic by-products which could otherwise adversely affect muscle function ${ }^{30}$. By maintaining or increasing blood flow, lactate can be redistributed to alternative sites such as the liver, heart and non-exercised muscles where it can be used as fuel or converted back to glucose and returned to the working muscles ${ }^{31,32}$. However, we found no significant differences in blood lactate concentrations in the conditions tested (BFR, PAS, NMESPlacebo and $\left.\mathrm{NMES}_{\mathrm{BF}+}\right)$ at any time point during the 30 min recovery period. This observation contradicts reports in other studies where blood lactate was found to be more effectively cleared when NMES was applied during recovery ${ }^{17,31,32}$. In particular, NMES was reported to improve the clearance of blood lactate following 200 yards swimming ${ }^{32}$ or exhausting climbing exercise ${ }^{31}$, although these studies did not test for an associated effect on performance recovery. The lack of difference in blood lactate levels between $\mathrm{NMES}_{\mathrm{BF}+}$ and other recoveries in the present study could be explained by the location to which NMES was applied. To induce a systemic approach, the NMES electrodes were applied to the calf muscles in our study, since these muscles generate most of the venous return. The capacity of NMES to speed blood lactate removal compared with passive rest reported elsewhere could be explained primarily by the increase of lactate release from the exercising muscles. Indeed, Bangsbo, et al. ${ }^{33}$ demonstrated that lactate uptake by inactive muscles is higher when local blood flow is increased by active recovery. Thus, applying a systemic treatment may decrease the effectiveness with which lactate is release from the active muscle during exercise. Authors ${ }^{17,31,32}$ tend to confirm this suggestion, as the muscles exposed to NMES during their recovery interventions tended to be those mainly mobilized during the exercise performed. Since the quadriceps were heavily 
"Relationship Between Blood Flow and Performance Recovery: A Randomized, Placebo-Controlled Study"

by Borne R, Hausswirth C, Bieuzen F

International Journal of Sports Physiology and Performance

(C) 2016 Human Kinetics, Inc.

involved in this exercise protocol, applying electrodes on this large muscle group could have improved lactate kinetics during recovery. However, the data of this present study do not allow to determine more precisely the mechanism by which lactate is removed, depending on the limb blood flow level. Further investigations are needed.

The second effect expected from the increase in blood flow induced by NMES during recovery can be partly explained by the muscle pump theory. Indeed, the muscular contraction reduces venous pressure within the active muscle, thus increasing the arteriovenous pressure gradient. One-way flow is ensured in the veins via one-way valves; the enhanced arteriovenous pressure gradient therefore results in an increase in arterial blood reaching the skeletal muscles $^{12}$. Because of this increase in arterial blood flow, NMES could increase oxygen supply to the exercised muscles ${ }^{32}$ and glucose uptake by the muscle cells ${ }^{34}$. For more than one hour after intense exercise, both pulmonary and muscle oxygen uptake can remain above resting levels ${ }^{35}$. This increase in oxygen demand is related to reloading of myoglobin and hemoglobin, resynthesis of ATP, PCr and, to a lesser extent, resynthesis of glycogen from lactate and other glycolytic intermediates. These findings support an important role for aerobic metabolism during repeated high-intensity exercises. Moreover, elevated blood flow during recovery increases glucose delivery to the muscles and its uptake, thus promoting glycogen synthesis ${ }^{36}$. Indeed, Bangsbo, et al. ${ }^{33}$ attributed a large percentage of muscle glycogen resynthesis to glucose uptake after short-term, high-intensity exercise. Furthermore, high-intensity exercise promotes maintenance of a high glucose level as a substrate for glycogen resynthesis, particularly in the early phase of post-exercise recovery ${ }^{37}$.

Finally, $\mathrm{NMES}_{\mathrm{BF}+}$ recovery intervention is thought to improve perceived recovery, this would explain how high-intensity performance is restored during a short-term recovery period $^{28,38,39}$. No other studies have fully explored the psychological effects of using NMES on performance parameters. Thus, it is not known whether information relating to techniques used 
"Relationship Between Blood Flow and Performance Recovery: A Randomized, Placebo-Controlled Study"

by Borne R, Hausswirth C, Bieuzen F

International Journal of Sports Physiology and Performance

(C) 2016 Human Kinetics, Inc.

during recovery could affect an athlete's pacing strategy in high-intensity short-duration exercise and/or whether pacing could be indicated before the exercise due to an effect on perceived recovery. The results presented in this paper, reporting no placebo effect on either performance recovery or perceived recovery, tend to indicate that the effects of NMES are not purely due to a placebo effect. These findings suggest that the physiological role of NMESinduced blood flow in recovery could be greater than the hypothesized psychological role.

\section{Conclusions and practical applications}

The novel finding in the present study is the demonstration of an effective, positive correlation between an increase in limb blood flow and performance recovery between bouts of high-intensity exercise. In comparison to traditional active recovery, this strategy presents the benefit to potentially preserve the organism of a possible energy and thermal overload, compromising the return to homeostasis. At the same time, this simple method can be easily included in a recovery routine, in sporting events inducing short recovery periods, during halftime in team sports and for sports with successive rounds. In particular, NMES-induced blood flow can be mixed with other recovery modalities, such as hydration and nutritional strategies, in the first moments following exercise when the time available for recovery is crucial but limited. Additional work utilizing NMES-induced blood flow during shorter or longer recovery periods and other exercise modes could elucidate the relative importance of the blood flow modulation in improving exercise performance. 
"Relationship Between Blood Flow and Performance Recovery: A Randomized, Placebo-Controlled Study" by Borne R, Hausswirth C, Bieuzen F

International Journal of Sports Physiology and Performance

(C) 2016 Human Kinetics, Inc.

\section{Acknowledgments}

The authors wish to thank the study participants for their commitment and cooperation.

\section{Conflict of interest}

The authors report no external sources of funding for this study. The authors report no conflicts of interest that are directly relevant to the content of this manuscript. 
"Relationship Between Blood Flow and Performance Recovery: A Randomized, Placebo-Controlled Study" by Borne R, Hausswirth C, Bieuzen F

International Journal of Sports Physiology and Performance

(C) 2016 Human Kinetics, Inc.

\section{References}

1. Barnett A. Using recovery modalities between training sessions in elite athletes: does it help? Sports Med 2006;36:781-796.

2. Minett GM, Costello JT. Specificity and context in post-exercise recovery: it is not a onesize-fits-all approach. Front Physiol 2015;6:130.

3. Nedelec M, Halson S, Abaidia AE, Ahmaidi S, Dupont G. Stress, Sleep and Recovery in Elite Soccer: A Critical Review of the Literature. Sports Med 2015;45:1387-1400.

4. Hinds T, McEwan I, Perkes J, Dawson E, Ball D, George K. Effects of massage on limb and skin blood flow after quadriceps exercise. Med Sci Sports Exerc 2004;36:13081313.

5. Fiscus KA, Kaminski TW, Powers ME. Changes in lower-leg blood flow during warm-, cold-, and contrast-water therapy. Arch Phys Med Rehabil 2005;86:1404-1410.

6. Vaile J, O'Hagan C, Stefanovic B, Walker M, Gill N, Askew CD. Effect of cold water immersion on repeated cycling performance and limb blood flow. Br J Sports Med 2011;45:825-829.

7. MacRae BA, Cotter JD, Laing RM. Compression garments and exercise: garment considerations, physiology and performance. Sports Med 2011;41:815-843.

8. Malone JK, Coughlan GF, Crowe L, Gissane GC, Caulfield B. The physiological effects of low-intensity neuromuscular electrical stimulation (NMES) on short-term recovery from supra-maximal exercise bouts in male triathletes. Eur J Appl Physiol 2012;112:2421-2432.

9. Korthuis RJ. Skeletal Muscle Circulation. San Rafael (CA), 2011.

10. Malone JK, Blake C, Caulfield BM. Neuromuscular electrical stimulation during recovery from exercise: a systematic review. J Strength Cond Res 2014;28:24782506.

11. Faghri PD, Votto JJ, Hovorka CF. Venous hemodynamics of the lower extremities in response to electrical stimulation. Arch Phys Med Rehabil 1998;79:842-848.

12. Valic Z, Buckwalter JB, Clifford PS. Muscle blood flow response to contraction: influence of venous pressure. J Appl Physiol (1985) 2005;98:72-76.

13. Grunovas A, Silinskas V, Poderys J, Trinkunas E. Peripheral and systemic circulation after local dynamic exercise and recovery using passive foot movement and electrostimulation. J Sports Med Phys Fitness 2007;47:335-343.

14. Griffin MB, Nicolaides AN, Bond D, Geroulakos G, Kalodiki E. The Efficacy of New VEINOPLUS Stimulation Technology to Increase Venous Flow and Prevent Venous Stasis. Journal of vascular surgery : official publication, the Society for Vascular Surgery [and] International Society for Cardiovascular Surgery, North American Chapter 2010;51:790. 
"Relationship Between Blood Flow and Performance Recovery: A Randomized, Placebo-Controlled Study"

by Borne R, Hausswirth C, Bieuzen F

International Journal of Sports Physiology and Performance

(C) 2016 Human Kinetics, Inc.

15. Abraham P, Mateus V, Bieuzen F, Ouedraogo N, Cisse F, Leftheriotis G. Calf muscle stimulation with the Veinoplus device results in a significant increase in lower limb inflow without generating limb ischemia or pain in patients with peripheral artery disease. J Vasc Surg 2013;57:714-719.

16. Miller BF, Gruben KG, Morgan BJ. Circulatory responses to voluntary and electrically induced muscle contractions in humans. Phys Ther 2000;80:53-60.

17. Bieuzen F, Borne R, Toussaint JF, Hausswirth C. Positive effect of specific lowfrequency electrical stimulation during short-term recovery on subsequent highintensity exercise. Appl Physiol Nutr Metab 2014;39:202-210.

18. Fairchild TJ, Armstrong AA, Rao A, Liu H, Lawrence S, Fournier PA. Glycogen synthesis in muscle fibers during active recovery from intense exercise. Med Sci Sports Exerc 2003;35:595-602.

19. Borne R, Hausswirth C, Costello JT, Bieuzen F. Low-frequency electrical stimulation combined with a cooling vest improves recovery of elite kayakers following a simulated 1000-m race in a hot environment. Scand J Med Sci Sports 2015;25 Suppl $1: 219-228$.

20. Beaven CM, Cook C, Gray D, Downes P, Murphy I, Drawer S, Ingram JR, Kilduff LP, Gill N. Electrostimulation's enhancement of recovery during a rugby preseason. Int J Sports Physiol Perform 2013;8:92-98.

21. Bieuzen F, Pournot H, Roulland R, Hausswirth C. Recovery after high-intensity intermittent exercise in elite soccer players using VEINOPLUS sport technology for blood-flow stimulation. J Athl Train 2012;47:498-506.

22. Finberg M, Braham R, Goodman C, Gregory P, Peeling P. Effects of electrostimulation therapy on recovery from acute team-sport activity. Int J Sports Physiol Perform 2013;8:293-299.

23. Broatch JR, Petersen A, Bishop DJ. Postexercise cold water immersion benefits are not greater than the placebo effect. Med Sci Sports Exerc 2014;46:2139-2147.

24. Beedie CJ, Foad AJ. The placebo effect in sports performance: a brief review. Sports Med 2009;39:313-329.

25. Klimek AT, Lubkowska A, Szygula Z, Chudecka M, Fraczek B. Influence of the ten sessions of the whole body cryostimulation on aerobic and anaerobic capacity. Int $\mathbf{J}$ Occup Med Environ Health 2010;23:181-189.

26. Cywinski J. May 8, 2012 2012. United States.

27. Takarada Y, Takazawa H, Sato Y, Takebayashi S, Tanaka Y, Ishii N. Effects of resistance exercise combined with moderate vascular occlusion on muscular function in humans. J Appl Physiol (1985) 2000;88:2097-2106.

28. Cortis C, Tessitore A, D'Artibale E, Meeusen R, Capranica L. Effects of post-exercise recovery interventions on physiological, psychological, and performance parameters. Int J Sports Med 2010;31:327-335. 
"Relationship Between Blood Flow and Performance Recovery: A Randomized, Placebo-Controlled Study"

by Borne R, Hausswirth C, Bieuzen F

International Journal of Sports Physiology and Performance

(C) 2016 Human Kinetics, Inc.

29. Hopkins WG, Marshall SW, Batterham AM, Hanin J. Progressive statistics for studies in sports medicine and exercise science. Med Sci Sports Exerc 2009;41:3-13.

30. Allen DG, Lamb GD, Westerblad H. Skeletal muscle fatigue: cellular mechanisms. Physiol Rev 2008;88:287-332.

31. Heyman E, B DEG, Mertens I, Meeusen R. Effects of four recovery methods on repeated maximal rock climbing performance. Med Sci Sports Exerc 2009;41:1303-1310.

32. Neric FB, Beam WC, Brown LE, Wiersma LD. Comparison of swim recovery and muscle stimulation on lactate removal after sprint swimming. J Strength Cond Res 2009;23:2560-2567.

33. Bangsbo J, Graham T, Johansen L, Saltin B. Muscle lactate metabolism in recovery from intense exhaustive exercise: impact of light exercise. J Appl Physiol (1985) 1994;77:1890-1895.

34. Henriksson J, Knol M. A single bout of exercise is followed by a prolonged decrease in the interstitial glucose concentration in skeletal muscle. Acta Physiol Scand 2005; 185:313-320.

35. Bangsbo J, Gollnick PD, Graham TE, Saltin B. Substrates for muscle glycogen synthesis in recovery from intense exercise in man. J Physiol 1991;434:423-440.

36. Bangsbo J, Hellsten Y. Muscle blood flow and oxygen uptake in recovery from exercise. Acta Physiol Scand 1998;162:305-312.

37. Pascoe DD, Gladden LB. Muscle glycogen resynthesis after short term, high intensity exercise and resistance exercise. Sports Med 1996;21:98-118.

38. Tessitore A, Meeusen R, Pagano R, Benvenuti C, Tiberi M, Capranica L. Effectiveness of active versus passive recovery strategies after futsal games. J Strength Cond Res 2008;22:1402-1412.

39. Warren CD, Brown LE, Landers MR, Stahura KA. Effect of three different betweeninning recovery methods on baseball pitching performance. J Strength Cond Res 2011;25:683-688. 
"Relationship Between Blood Flow and Performance Recovery: A Randomized, Placebo-Controlled Study" by Borne R, Hausswirth C, Bieuzen F

International Journal of Sports Physiology and Performance

(C) 2016 Human Kinetics, Inc.

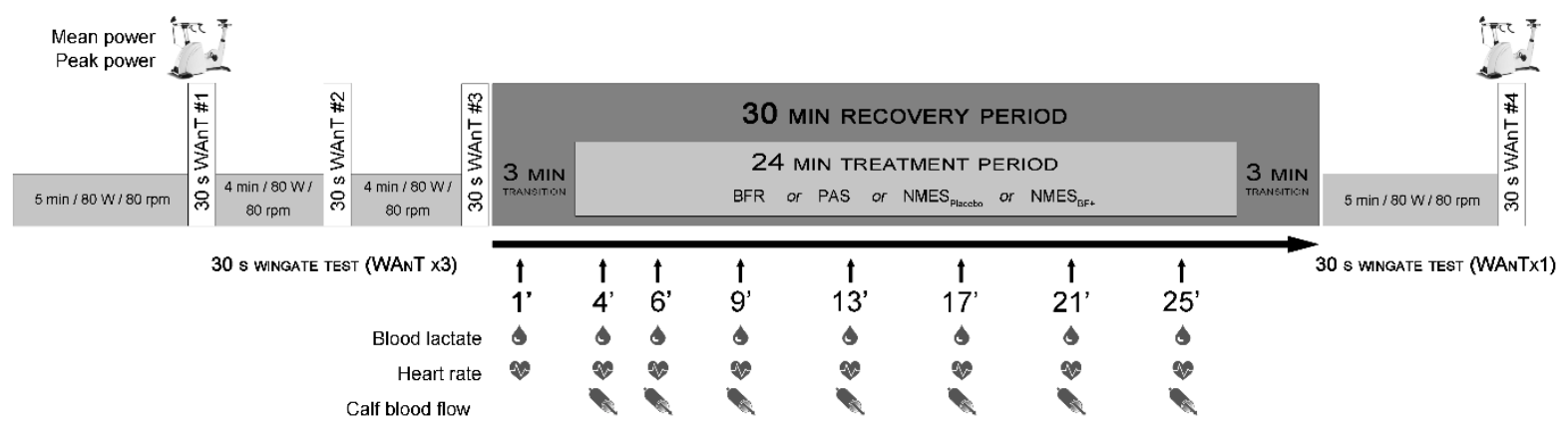

Figure 1. Study design. WAnT, Wingate test; BFR, Blood flow restriction; PAS, passive; NMES $S_{\text {Placebo, }}$ placebo; $\mathrm{NMES}_{\mathrm{BF}+}$, neuromuscular electrostimulation-induced blood flow. 
"Relationship Between Blood Flow and Performance Recovery: A Randomized, Placebo-Controlled Study" by Borne R, Hausswirth C, Bieuzen F

International Journal of Sports Physiology and Performance

(C) 2016 Human Kinetics, Inc.
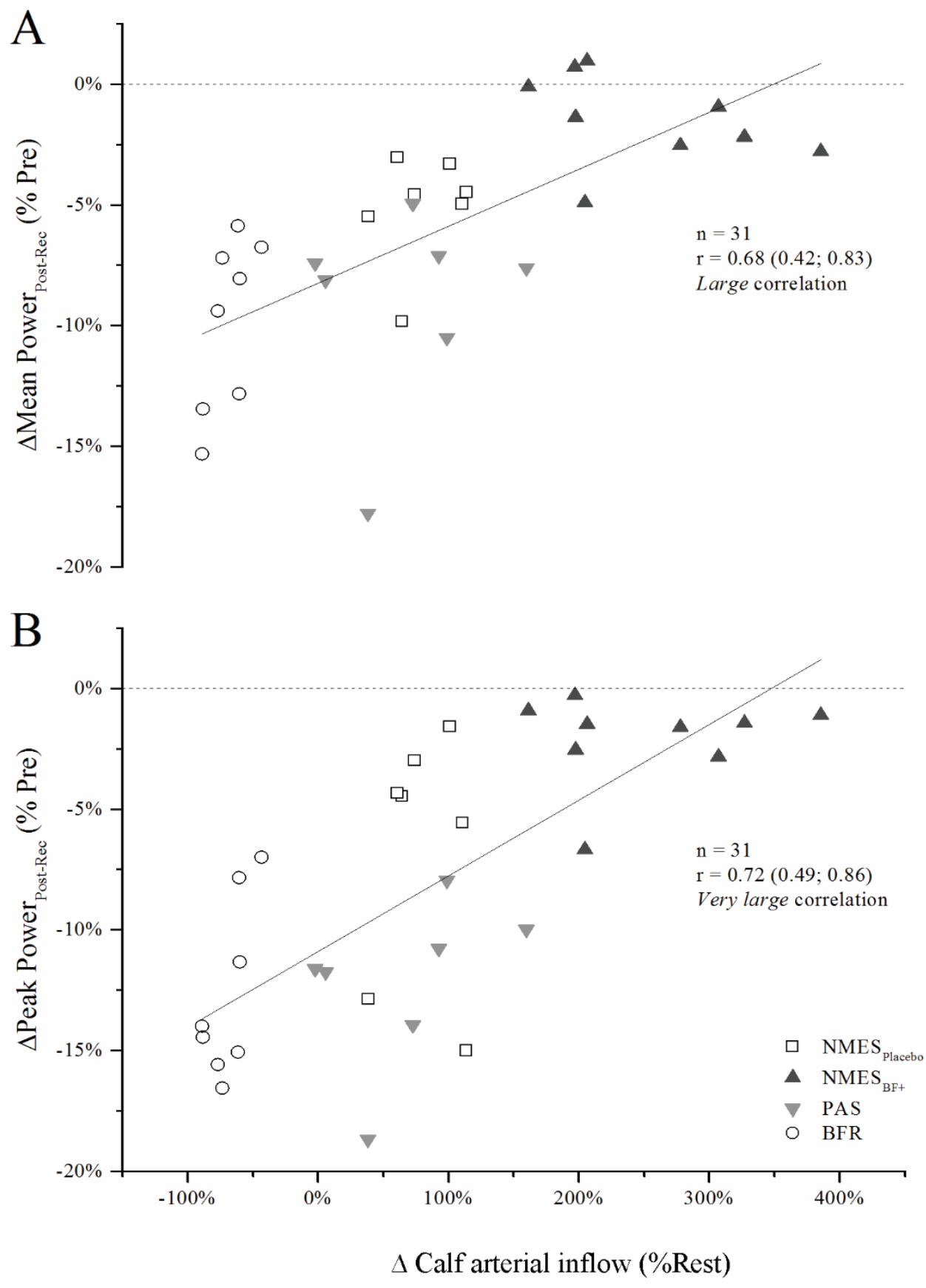

Figure 2. Relationship between the change in Mean PowerPost-Rec (A) and Peak Power Post-Rec (B) output and the change in calf arterial inflow following either blood flow restriction (BFR, $\bigcirc$ ), passive (PAS, $\nabla$ ), placebo (NMESPlacebo, $\square$ ) and neuromuscular electrostimulationinduced blood flow $\left(\mathrm{NMES}_{\mathrm{BF}+}, \mathbf{\Delta}\right)$ recovery interventions. 
"Relationship Between Blood Flow and Performance Recovery: A Randomized, Placebo-Controlled Study" by Borne R, Hausswirth C, Bieuzen F

International Journal of Sports Physiology and Performance

(C) 2016 Human Kinetics, Inc.
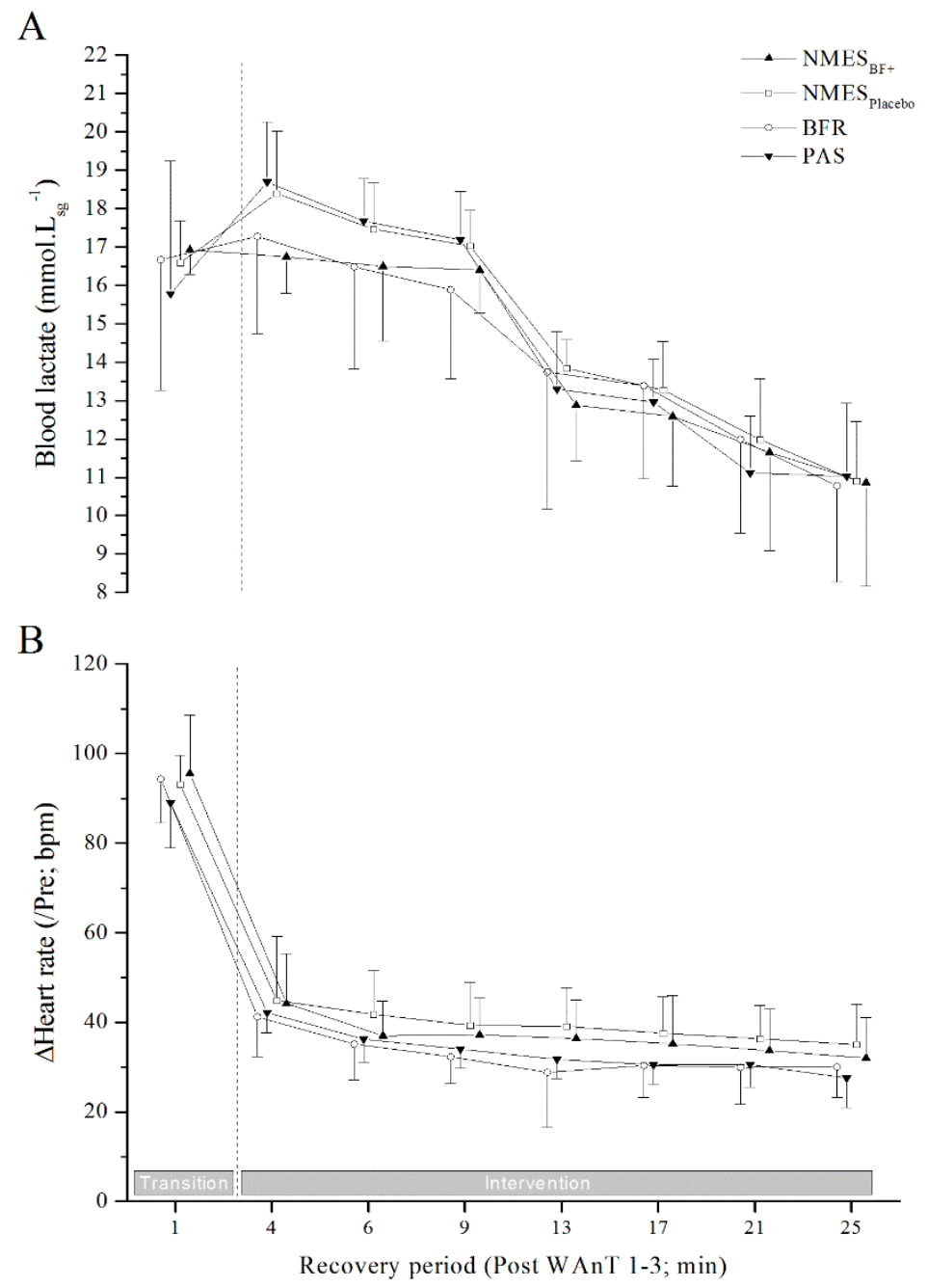

Figure 3. Blood lactate concentration (A) and changes in heart rate from rest (B) (mean \pm SD) during blood flow restriction (BFR, $\bigcirc$ ), passive (PAS, $\boldsymbol{\nabla}$ ), placebo (NMESPlacebo, $\square$ ) and neuromuscular electrostimulation-induced blood flow $\left(\mathrm{NMES}_{\mathrm{BF}+}, \boldsymbol{\Delta}\right)$ interventions throughout the recovery period. No significant differences between the four recovery intervention protocols. 
"Relationship Between Blood Flow and Performance Recovery: A Randomized, Placebo-Controlled Study" by Borne R, Hausswirth C, Bieuzen F

International Journal of Sports Physiology and Performance

(C) 2016 Human Kinetics, Inc.

Table 1. Participant physical characteristics (mean \pm SD).

\begin{tabular}{ccccc}
\hline & NMES $_{\mathbf{B F +}}$ & NMES $_{\text {Placebo }}$ & BFR & PAS \\
\hline Number & $\mathrm{n}=10$ & $\mathrm{n}=8$ & $\mathrm{n}=8$ & $\mathrm{n}=7$ \\
& {$[\hat{\jmath}=8, \uparrow=2]$} & {$[\hat{\jmath}=6, \uparrow=2]$} & {$[\hat{\jmath}=8]$} & {$[\hat{\jmath}=7]$} \\
Age (years) & $30 \pm 5$ & $23 \pm 4$ & $30 \pm 7$ & $24 \pm 3$ \\
Height (cm) & $173 \pm 8$ & $173 \pm 5$ & $177 \pm 6$ & $181 \pm 3$ \\
Body mass (kg) & $69 \pm 12$ & $71 \pm 15$ & $70 \pm 8$ & $74 \pm 5$ \\
$\begin{array}{c}\text { Body fat (6 sites) (\%) } \\
\text { Training hour per } \\
\text { week (h) }\end{array}$ & $9.9 \pm 2.4$ & $9.8 \pm 2.3$ & $9.2 \pm 2.1$ & $9.7 \pm 2.5$ \\
\hline
\end{tabular}


Table 2. Sum of the total work measured during the first Wingate anaerobic test session (WAnT 1-3). Mean power (MPO) and peak power (PPO) values of the first (WAnT 1) and the fourth (WAnT 4) Wingate test and variation of calf arterial inflow between rest and recovery intervention protocol following neuromuscular electrostimulation-induced blood flow (NMES $\mathrm{BF}$ ), placebo (NMES placebo), blood flow restriction (BFR) and passive recovery (PAS) interventions. Values are expressed in mean \pm SD.

\begin{tabular}{|c|c|c|c|c|c|c|c|c|c|c|c|c|c|}
\hline \multirow[b]{2}{*}{ Total Work $\left(\mathrm{KJ} ; \mathbf{1 0}^{3}\right)$} & \multirow[b]{2}{*}{$\sum$ WAnT 1-3 } & \multicolumn{3}{|c|}{ NMES $_{\mathrm{BF}+}{ }^{\mathrm{s}}$} & \multicolumn{3}{|c|}{ NMES $_{\text {Placebo }}$} & \multicolumn{3}{|c|}{ BFR $^{\mathrm{S}}$} & \multicolumn{3}{|c|}{ PAS } \\
\hline & & 51.7 & \pm & 13.5 & 51.3 & \pm & 7.8 & 51.6 & \pm & 2.2 & 52.0 & \pm & 12.4 \\
\hline \multirow{2}{*}{$\operatorname{PPO}(W)$} & WAnT 1 & 793 & \pm & 140 & 822 & \pm & 201 & 857 & \pm & 133 & 843 & \pm & 59 \\
\hline & WAnT 4 & 774 & \pm & 125 & 759 & \pm & $139^{\#}$ & 746 & \pm & $98^{*}$ & 741 & \pm & $64^{*}$ \\
\hline \multirow{2}{*}{ MPO (W) } & WAnT 1 & 687 & \pm & 118 & 630 & \pm & 109 & 660 & \pm & 68 & 698 & \pm & 61 \\
\hline & WAnT 4 & 676 & \pm & 106 & 598 & \pm & $106^{\#}$ & 594 & \pm & $52 *$ & 634 & \pm & $60^{*}$ \\
\hline \multirow{3}{*}{ BF (\% Pre) } & 4-9 min & 249 & \pm & 86 & 75 & \pm & 52 & -72 & \pm & 17 & 75 & \pm & 52 \\
\hline & $13-17 \mathrm{~min}$ & 230 & \pm & 73 & 68 & \pm & 41 & -68 & \pm & 15 & 65 & \pm & 66 \\
\hline & $21-25 \min$ & 195 & \pm & $71^{\#}$ & 52 & \pm & 39 & -64 & \pm & 18 & 39 & \pm & 28 \\
\hline
\end{tabular}

*, Represents a significant difference from WAnT 1 ( $<<0.001)$; \#, represents a significant difference from WAnT $1(\mathrm{p}<0.05)$.

$\$$, Represent a significant difference in calf arterial inflow from other interventions at each time point $(\mathrm{P}<0.001)$.

\#, Represent a significant difference in calf arterial inflow from the 4-9 min measurement period $(\mathrm{P}<0.001)$. 
"Relationship Between Blood Flow and Performance Recovery: A Randomized, Placebo-Controlled Study"

by Borne R, Hausswirth C, Bieuzen F

International Journal of Sports Physiology and Performance

(C) 2016 Human Kinetics, Inc.

Table 3. Perceptions of recovery (efficacy and well being) responses (mean \pm SD) following neuromuscular electrostimulation-induced blood flow $\left(\mathrm{NMES}_{\mathrm{BF}+}\right)$, placebo (NMES Placebo), blood flow restriction (BFR) and passive recovery (PAS) interventions. No significant differences between the four recovery intervention protocols.

\begin{tabular}{ccccc}
\hline & NMES $_{\text {BF }+}$ & NMES $_{\text {Placebo }}$ & BFR & PAS \\
\hline $\begin{array}{c}\text { Recovery } \\
\text { efficacy }\end{array}$ & $7.2 \pm 1.9$ & $5.8 \pm 1.9$ & $5.7 \pm 1.4$ & $5.4 \pm 2.4$ \\
$\begin{array}{c}\text { Recovery } \\
\text { well-being }\end{array}$ & $7.3 \pm 2.0$ & $5.7 \pm 3.5$ & $5.9 \pm 2.5$ & $5.4 \pm 2.6$ \\
\hline
\end{tabular}

